DESY $06-121$

\title{
Two Loop QFT in the Making
}

\author{
Stefano Actis ${ }^{\mathrm{a}}$, Giampiero Passarino ${ }^{\mathrm{b}}$, Sandro Uccirati ${ }^{\mathrm{b}}$ \\ ${ }^{a}$ Deutsches Elektronen-Synchrotron, Zeuthen, Germany \\ ${ }^{\mathrm{b}}$ Dipartimento di Fisica Teorica, Università di Torino, Italy \\ INFN, Sezione di Torino, Italy
}

Recent developments in the evaluation of two-loop pseudo-observables and observables are briefly reviewed.

\section{Introduction}

The complete strategy to derive theoretical predictions for pseudo-observables (PO) and observables $(\mathrm{O})$ up to two-loop accuracy is based on the following steps: generation and manipulation of diagrams, renormalization, semi-numerical evaluation of diagrams. We perform the first step with the help of the code $\mathcal{G}$ raphShot (FORM 3.1) [1] while the last is performed using the code $\mathcal{L}$ oop Back (FORTRAN 95) [2] which makes extensive use of array handling, assignment overloading, vector/recursive functions.

\section{Renormalization and Unitarity}

A keyword in renormalization is counterterm; they are not strictly needed but are, nevertheless, very useful when dealing with overlapping divergencies. GraphShot generates all counterterms needed in the standard model and produces ultraviolet (UV) finite Green functions. Another important keyword is skeleton expansion, meaning that the relevant objects in perturbation theory are dressed propagators. With their help we want to construct a (finite) renormalization procedure where a) the renormalized parameters are real, b) finite renormalization is the result of a consistently truncated solution of renormalization equations (RE), c) complex poles arise naturally after dressing the propagators, but cutting equations remain valid to all orders. For an unstable particle $V$ define

$\bar{\Delta}_{V}=\frac{\Delta_{V}}{1-\Delta_{V} \Sigma_{V V}}$, where $\Sigma$ is the $V$ (skeleton) self-energy. Cuttingequations and unitarity of the $S$-matrix can be proven: one uses two-loop $\bar{\Delta}$ in tree diagrams, one-loop $\bar{\Delta}$ in one-loop diagrams and tree propagators in two-loop diagrams. The proof is due to Veltman [3]: the crucial observation is that $\bar{\Delta}$ satisfies the Källen - Lehmann representation,

$\bar{\Delta}_{V}^{+}\left(p^{2}\right)=\theta\left(p_{0}\right)\left[\bar{\Delta}_{V}\left(p^{2}\right)\right]^{2} 2 \operatorname{Im} \Sigma_{V V}\left(p^{2}\right)$

while, for a stable particle $s$, the pole term shows up as

$$
\begin{aligned}
\bar{\Delta}_{s}^{+}\left(p^{2}\right) & =\theta\left(p_{0}\right)\left[\bar{\Delta}_{s}\left(p^{2}\right)\right]^{2} 2 \operatorname{Im} \Sigma_{s s}\left(p^{2}\right) \\
& +2 i \pi \delta\left(p^{2}+m_{s}^{2}\right) .
\end{aligned}
$$

One then expresses $\operatorname{Im} \Sigma_{V V}$ in terms of cut selfenergies, repeats ad libidum and derives that contributions from cut lines come from stable particles only. Consider a toy model with

$L_{\mathrm{int}}=\frac{g}{2} \Phi(x) \phi^{2}(x)$,

and where $\Phi$ is unstable. We define $\bar{\Delta}_{\Phi}$ and $\bar{\Delta}_{\phi}$ according to Eq.(11). An example of the skeleton expansion for self-energies is given in Fig. 1: $\operatorname{Im} \Sigma_{\phi \phi} \neq 0$ only due to the 3 -particle cut of diagram b) of Fig. 1 and only diagrams a) and c) are retained in the expansion; in a) we use $\bar{\Delta}_{\Phi}$, at one-loop accuracy.

In a gauge theory, however, there is a clash between resummation and gauge invariance; usually only the complex pole is resummed [4]. Therefore a one-loop self-energy with $\bar{\Delta}_{\Phi}$ at one-loop 
accuracy is equivalent to the 3 diagrams of Fig. 2 computed with $\Delta_{\Phi}\left(s_{M}\right)$, where

$Z_{p}=\frac{g^{2}}{16 \pi^{2}} B_{0}\left(-s_{M} ; m, m\right)$.

The interplay of gauge parameter independence, Ward - Slavnov - Taylor identities and unitarity (which is naturally satisfied in the framework of dressed propagators) requires a more detailed analysis, beyond the scope of this paper.

To solve REs we need an input parameter set (IPS) including some notion of $M_{\text {exp }}$. In the past on-sell PO have been derived by fitting lineshapes from experiments [ [5] but we can use an on-shell $M_{O S}$ only at one-loop. Beyond this order the correct treatment requires introducing complex poles [ [], $s_{V}=\mu_{V}^{2}-i \gamma_{V} \mu_{V}$.

If we want to use available data a transformation is therefore needed: define pole PO through $\psi=\arctan \Gamma_{O S} / M_{O S}: M_{P}=M_{O S} \cos \psi$ and $\Gamma_{P}=\Gamma_{O S} \sin \psi$. Once again, a change of strategy is needed since $\mathrm{RE}$ change their structure at two loops. It is a new perspective: at one loop one considers $M_{O S}$ as an input parameter independent of $s_{P}$ and derive $s_{P}$. At two loop REs are written for real $p_{R}$ and solved in terms of (among other things) experimental $s_{P}$.

In the framework of an order-by-order renormalization, $M_{R}$ is a real solutions of truncated REs consistently with cutting-equations and unitarity.

\subsection{Complex poles}

Here we give an example of the old fashioned one-loop technique: on-shell masses are input and, having the Higgs boson in mind, we derive $s_{H}=\mu_{H}^{2}-i \mu_{H} \gamma_{H}$; numerical results for $s_{H}$ are shown in Tab. 1

The current fashion instead is to extract $s_{H}^{\exp }=$ $\mu_{H}^{2}-i \mu_{H} \gamma_{H}$ from data and to derive $s_{H}^{\text {th }}=M_{H}^{2}-$ $i M_{H} \Gamma_{H}$. To see how it works we point out the difference with previous two-loop calculations [ [7] where one starts from

$s_{V}=m^{2}-\Sigma_{V V}\left(s_{V}, m^{2}, \ldots\right)$,

and derives the complex pole in terms of the (bare) renormalized mass

$s_{V}=m^{2}-\Sigma_{V V}^{(1)}\left(m^{2}, m^{2}, \ldots\right)+\ldots$
Table 1

One-loop $s_{H}\left(M_{H}^{O S}\right)$

\begin{tabular}{|l|l|l|}
\hline$M_{H}^{O S}[\mathrm{GeV}]$ & 120 & 300 \\
& & \\
\hline$\mu_{H}[\mathrm{GeV}]$ & 119.96 & 299.74 \\
\hline$\gamma_{H}[\mathrm{GeV}]$ & $7.00 \times 10^{-3}$ & 7.90 \\
\hline
\end{tabular}

To improve the quality of the result we consider the relation

$s_{V}=m^{2}-\Sigma_{V V}\left(s_{V}, m^{2},\{p\}, \ldots\right)$

where with $\{p\}$ we denote additional, renormalized, parameters. We then solve REs and obtain $m^{2}$ and $\{p\}$, i.e.

$m^{2},\{p\}=\operatorname{Re} f\left(s_{V_{1}}, s_{V_{2}}, \ldots\right)$,

where $s_{V_{i}}$ are experimental complex poles. Note that we never expand functions depending on complex POs which means that we actually compute two-loop diagrams on the second Riemann sheet. Furthermore, in our scheme the solution of REs is used at the Born level in two-loop diagrams, one-loop in one-loop diagrams, two-loop in tree diagrams. If $V \notin\left\{V_{1}, V_{2}, \ldots\right\}$ we have a genuine prediction, otherwise we have a consistency relation for loop corrections. Numerical results for the two-loop $s_{H}^{\text {th }}\left(s_{H}^{\exp }\right)$ are shown in Tab. 2

Table 2

Two-loop $s_{H}^{\text {th }}\left(s_{H}^{\exp }\right)$. All entries in GeV.

\begin{tabular}{|l|l|l|l|}
\hline$\mu_{H}$ & $\gamma_{H}$ & $M_{H}$ & $\Gamma_{H}$ \\
\hline 300 & 4 & 299.96 & 8.374 \\
\hline 300 & 12 & 299.87 & 8.376 \\
\hline 500 & 40 & 500.17 & 63.37 \\
\hline 500 & 80 & 500.42 & 63.34 \\
\hline
\end{tabular}


Table 3

Percentage two-loop corrections in RE Eq.(10).

\begin{tabular}{|l|l|l|l|}
\hline$M_{H}^{O S}[\mathrm{GeV}]$ & 150 & 300 & 500 \\
\hline$\frac{G_{F} \mu_{W}^{2}}{2 \pi^{2}} \frac{\delta_{G}^{(2)}}{\delta_{G}^{(1)}}$ & $18.29 \%$ & $8.89 \%$ & $-24.62 \%$ \\
\hline
\end{tabular}

\subsection{Numbers \& renormalization}

Consider one of the REs, e.g.

$\frac{G_{F}}{\sqrt{2}}=\frac{g^{2}}{8 M^{2}}(1+\Delta g), \quad \Delta g=\delta_{G}+\Delta g^{S}$,

relating $g$ to the Fermi constant $G_{F} ; \Delta g^{S}$ is the $W$ self-energy part. A solution in perturbation theory starts with

$$
\begin{aligned}
g^{2} & =8 G_{F} \mu_{W}^{2}\left[1+C_{g}^{(1)} \frac{G_{F}}{\pi^{2}}+\ldots\right], \\
C_{g}^{(1)} & =\frac{1}{2}\left[\operatorname{Re} \Sigma_{W W}^{(1)}\left(s_{W}\right)-\Sigma_{W W}^{(1)}(0)\right] .
\end{aligned}
$$

According to an old result $\delta_{G}^{(1)}$ is $\mathrm{UV} / \mathrm{IR}$ finite; we can add that $\delta_{G}^{(2)}$ is finite after one-loop renormalization. Furthermore, we define a process independent Fermi coupling at the two-loop level

$$
\begin{aligned}
G & =G_{F}\left\{1-\delta_{G}^{(1)} \frac{G_{F} \mu_{W}^{2}}{2 \pi^{2}}+\left[2\left(\delta_{G}^{(1)}\right)^{2}\right.\right. \\
& \left.\left.-\frac{2}{\mu_{W}^{2}} \delta_{G}^{(1)} C_{g}^{(1)}-\delta_{G}^{(2)}\right]\left(\frac{G_{F} \mu_{W}^{2}}{2 \pi^{2}}\right)^{2}\right\} .
\end{aligned}
$$

Therefore, starting from a RE like

$$
\begin{aligned}
X & =x\left(1+a_{1} x+a_{2} x^{2}\right), \\
X & =\frac{G_{F} \mu_{W}^{2}}{2 \pi^{2}}, \quad x=\frac{g^{2}}{16 \pi^{2}}, \quad a_{1}=\delta_{G}^{(1)}+S^{(1)}, \\
a_{2} & =S^{(1)}\left[\delta_{G}^{(1)}+S^{(1)}\right]+\delta_{G}^{(2)}+S^{(2)},
\end{aligned}
$$

we obtain the following solution:

$x=X+X^{2}\left(b_{1}+b_{2} X\right), \quad S^{(n)}=\frac{\Sigma_{W W}^{(n)}(0)}{\mu_{W}^{2}}$.

The LO/NLO/NNLO terms are $X, b_{1} X^{2}$ and $b_{2} X^{3}$; results are shown in Tabs. 3 , 4 where one can see that perturbation theory becomes questionable beyond $350 \mathrm{GeV}$.
Table 4

Percentage two-loop corrections in RE Eq.(14).

\begin{tabular}{|l|l|l|l|}
\hline$M_{H}^{O S}[\mathrm{GeV}]$ & 150 & 250 & 350 \\
\hline NLO/LO $(\%)$ & +3.31 & -2.30 & -7.85 \\
\hline$b_{1}$ & +12.28 & -8.51 & -29.07 \\
\hline$b_{2} X$ & +0.25 & -1.38 & -9.26 \\
\hline NNLO/NLO $(\%)$ & +2.06 & +16.16 & +31.85 \\
\hline
\end{tabular}

\section{Running of $\alpha$}

The role played by the running of $\alpha$ has been crucial in the development of precision tests of the SM. Underneath this concept there is the popular wisdom that universal corrections are the important ingredient while non-universal ones should be made as small as possible; therefore, universal corrections should be linked to a set of POs and data should be presented in the language of POs which, in turn, is connected with resummation, against gauge invariance. Admittedly, around $M_{Z}$ it has been easy to perform a discrimination, relevant vs. irrelevant terms, paying a little price to gauge invariance. Well above this scale the situation is drastically different. Thus, the natural question is about the definition of the running of $\alpha$ at an arbitrary scale. One (fuzzy) idea is to import from QCD the concept of $\overline{M S}$ couplings and to express theoretical predictions through $\overline{M S}$ couplings [ 8]. This idea is open for criticism: although the $\overline{M S}$ parameter seems unambiguous it violates decoupling.

There is another, well-known, solution: do the calculation in the $R_{\xi}$ gauge, select a $\xi$ - independent part of self-energies, perform resummation while leaving the rest to ensure independence when combined with vertices and boxes. The obvious criticism is: it violates uniqueness; however, it is only a matter of conventions.

Ingredients for $\alpha \overline{M S}$ are: a bosonic part, a fermionic part with 3 lepton generations, a perturbative quark contribution (top or diagrams where light quarks are coupled internally to mas- 
sive vector bosons) and a non-perturbative one with diagrams where a light quark couples to a photon (related to $\Delta \alpha_{\text {had }}^{5}\left(M_{Z}^{2}\right)$ ). We define

$\alpha_{\overline{M S}}^{-1}(s)=\alpha^{-1}-\left.\frac{1}{4 \pi} \Pi_{Q Q}^{\overline{M S}}(0)\right|_{\mu^{2}=s}$.

Alternatively, we consider $\xi=1$ and define

$\frac{\alpha}{\alpha(s)}=1+\Delta \alpha(s)$

Numerical results are shown in Tab. [5

\section{Virtual infrared corrections}

We have been able to prove [9] that two-loop vertices have an integral representation

$\int d C_{k}(\{x\}) \frac{1}{A}\left[\ln \left(1+\frac{A}{B}\right)\right.$ or $\left.\operatorname{Li}_{n}\left(\frac{A}{B}\right)\right]$,

where $A, B$ are multivariate polynomials in $\{x\}$, the Feynman parameters. Two - loop diagrams are always reducible to combinations of integrals of this type where the usual monomials that appear in the integral representation of Nielsen - Goncharov generalized polylogarithms are replaced by multivariate polynomials of arbitrary degree.

Fig. 3 Example of IR divergent two-loop vertex.

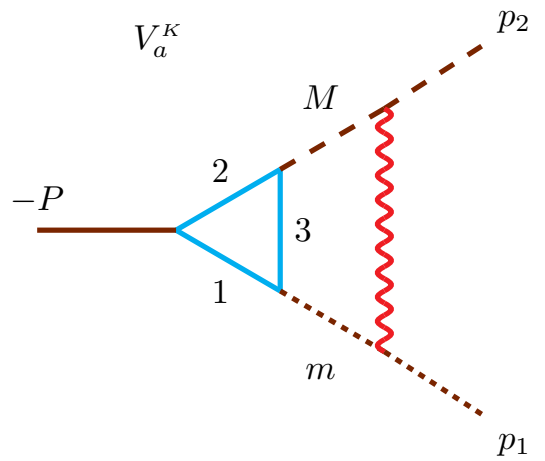

Our method is fully multi-scale, it allows for a classification of infrared divergent configurations, for the evaluation of IR residues and IR finite parts and is also suitable for collinear regions. To reach these objectives we had to extend Berstein Sato - Tkachov functional relations [11] to higher order transcendental functions. Results for the diagram in Fig. 3 are shown in Tab. [6

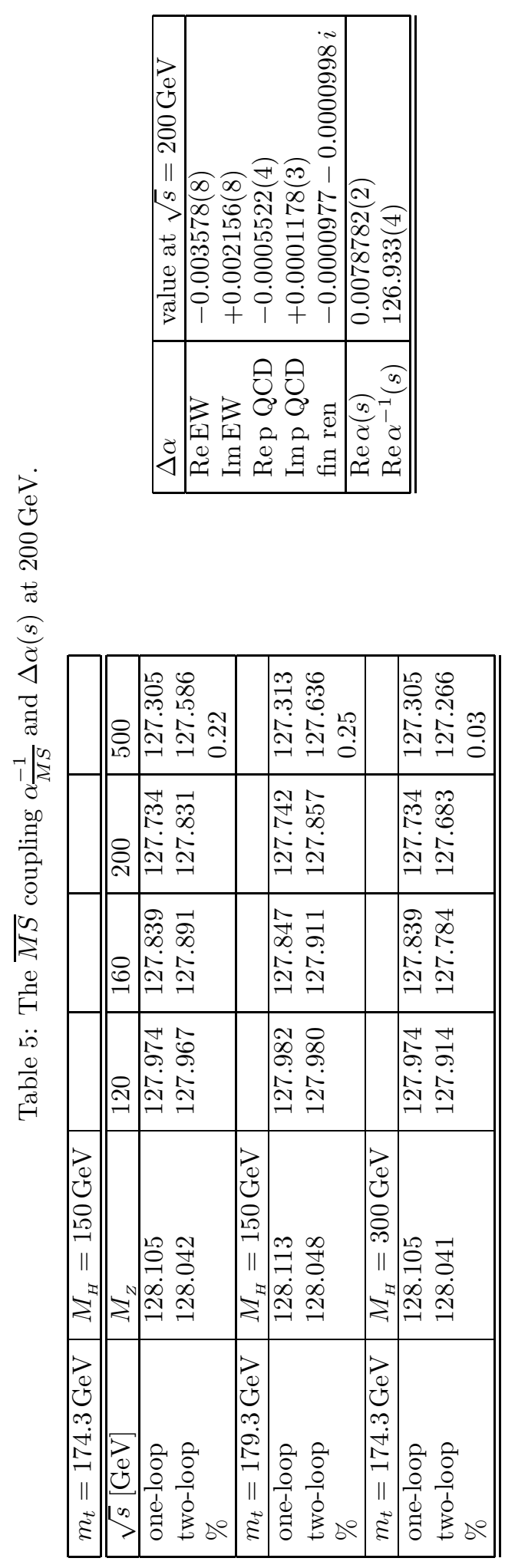


Table 6

Comparison with the results of Davydychev Kalmykov [ 10]. Only the infrared finite part is shown, in units of $10^{-8} \mathrm{GeV}^{-4}$.

\begin{tabular}{|l|c|l|l|}
\hline & $\sqrt{s}$ & $\operatorname{Re} V_{0 ; K}$ & $\operatorname{Im} V_{0 ; K}$ \\
\hline Our & 400 & $5.1343(1)$ & $1.94009(8)$ \\
DK & & 5.13445 & 1.94008 \\
\hline Our & 300 & 5.68801 & -1.61218 \\
DK & & 5.68801 & -1.61218 \\
\hline Our & 200 & 9.36340 & -2.84232 \\
DK & & 9.36340 & -2.84232 \\
\hline Our & 100 & 29.4726 & -9.74218 \\
DK & & 29.4726 & -9.74218 \\
\hline
\end{tabular}

\section{REFERENCES}

1. S. Actis, A. Ferroglia, G. Passarino, M. Passera and S. Uccirati, $\mathcal{G}$ raphShot, work in progress.

2. G. Passarino and S. Uccirati, Loop Back, work in progress.

3. M. J. G. Veltman, Physica 29, 186 (1963).

4. A. Denner, S. Dittmaier, M. Roth and L. H. Wieders, Nucl. Phys. B 724 (2005) 247 [arXiv hep-ph/0505042].

5. M. W. Grunewald et al., Four-fermion production in electron positron collisions, [arXiv hep-ph/0005309].

6. W. Beenakker et al., Nucl. Phys. B $\mathbf{5 0 0}$ (1997) 255 [arXiv hep-ph/9612260.

7. F. Jegerlehner, M. Y. Kalmykov and O. Veretin, Nucl. Instrum. Meth. A 502, 618 (2003).

8. G. Degrassi and A. Vicini, Phys. Rev. D 69, 073007 (2004) [arXiv hep-ph/0307122.

9. G. Passarino and S. Uccirati, Nucl. Phys. B 747 (2006) 113 [arXiv hep-ph/0603121.

10. A. I. Davydychev and M. Y. Kalmykov, Nucl. Phys. B 699 (2004) 3 [arXiv hep-th/0303162.

11. F. V. Tkachov, Nucl. Instrum. Meth. A 389 (1997) 309 [arXiv hep-ph/9609429; J. Bernstein, Functional Analysis and its Applications 5(1971); M. Sato, Nagoya Mat. J. 120 (1990) 1.
Figure 1. Example of skeleton expansion.

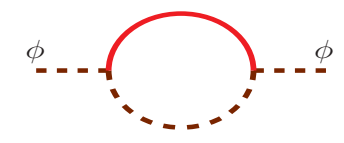

a) skeleton

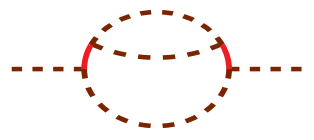

b) $\Sigma$ insertion

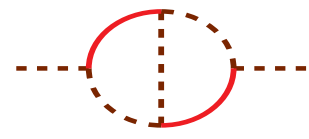

c) skeleton

Figure 2. Rearranging perturbation theory in the presence of complex poles.
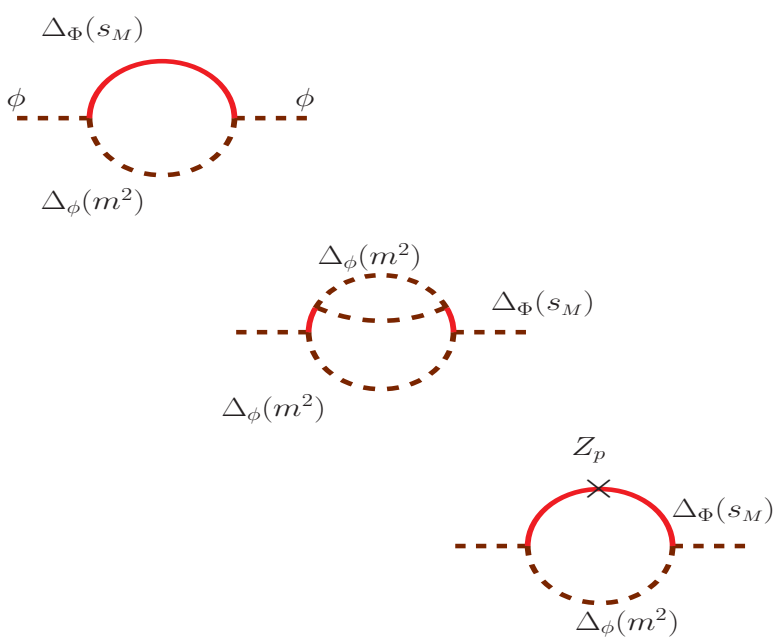